\title{
COMPUTATIONAL MAGNETOHYDRODYNAMIC MODEL OF A NONUNIFORM GASDYNAMIC MIRROR PROPULSION SYSTEM
}

\author{
Chad J. Ohlandt*, Dept. of Aerospace Engineering \\ Terry Kammash ${ }^{\dagger}$, Dept. of Nuclear Engineering \\ Kenneth G. Powell ${ }^{\ddagger}$, Dept. of Aerospace Engineering \\ University of Michigan, Ann Arbor, MI 48109
}

\begin{abstract}
Parametric engineering models of the gas dynamic mirror (GDM) fusion propulsion systems[1, 2, 3, 4] have produced potentially feasible systems. However, these systems are extremely large and massive due to neutron or bremsstrahlung radiation losses for traditional and advanced fusion fuels, respectively. In order to explore designs beyond the simple uniform GDM represented by the parametric model, a 3-D complex geometry ideal magnetohydrodynamic (MHD) modeling tool[5] based on finite volume methods and adaptive Cartesian grids was developed. The explicit nature of the algorithm combined with the near relativistic magneto-acoustic wave speeds limited the utility of the model. Presented here is a new MHD implicit alorithm with embedded magnetic fields which is capable of modeling complex, nonuniform GDMs.
\end{abstract}

\section{Introduction}

Previous studies[1, 2, 3, 4] of gas dynamic mirror (GDM) fusion propulsion systems for interplanetary exploration have demonstrated that they are feasible, see Figure 1. However, systems of large size (100-1000 meters) and mass (400-1000 tons) assembled in orbit are required. The resulting cost barriers restrict the practical development of GDM propulsion systems.

The need for such large systems is driven by neutron and radiation losses from the plasma. Losses need to be compensated for with longer systems to improve containment or hotter plasmas to increase reactivity. Of course, longer and hotter systems have greater energy losses, producing diminishing returns. Advanced fusion fuels with low neutron emmissions have been explored, but radiation losses from the much higher temperatures produce the same limitations[4]. Table 1 lists potential fusion fuels with important parameters.

Past studies have generally assumed a homogeneous plasma cylinder at the center of the tandem magnetic mirror confinement system. Nonuniform configurations of the plasma due to various injection and heating methods have the potential to reduce the size requirements for GDM systems. Unfortunately, nonuniform systems are complex and difficult to model.

A computational magnetohydrodynamic (MHD) model was constructed using full 3-D Cartesian adaptive grids and with finite volume MHD flow solvers[5]. To address the extremely large magnetic fields in the GDM system, the algorithm uses embedded magnetic fields to limit errors[6]. However, these large magnetic fields in GDMs also produced magneto-acoustic wave speeds approaching a few percent the speed of light. Combined with a grid that attempted to resolve down to the centimeter scale, this effectively drove the time step to zero in order to meet the CFL condition required by the explicit algorithm.

A new implicit flow solution that includes the magnetic source terms and embedded magnetic fields of the original model is described below. In order to addressed the added computational demand of the implicit algorithm, the code uses a parallel library, PETSci $[7,8,9]$, to solve the resulting system of equations.

\footnotetext{
${ }^{*}$ Graduate Student, Member AIAA

${ }^{\dagger}$ Professor, Associate Fellow AIAA

¥Professor, Member AIAA

Copyright (C) 2003 by Chad Ohlandt. Published by the American Institute of Aeronautics and Astronautics, Inc., with permis-
} sion. 


\section{Algorithm Review}

Beyond the implicit flow solver, the model is largely the same as the previous work. [5] The grid generator is a 3-D unstructured Cartesian grid stored in an octree format with solution-based refinement. Current test criteria for solution adaptation include velocity gradients $(\nabla \mathbf{v})$, flow vorticity $(\nabla \times \mathbf{v})$, numeric entropy wave strength $\left(\nabla p-a^{2} \nabla \rho\right)$, and current flux $(\nabla \times \mathbf{B})$. See Figure 2 for a sample solution adaptive grid.

The GDM is modeled using standard ideal magnetohydrodynamic equations. Continuum flow, nonrelativistic, neutral charge density, and inviscid assumptions produce a combination of the Euler equations with a vector magnetic equation, a system of eight equations. As in the previous work, we retain the divergence of the $\mathbf{B}$ field in the source terms to correct for computational error and to maintain the symmetrizable form. A form of the equations with embedded magnetic fields allows the accurate modeling of MHD flows in extremely high $\mathbf{B}$ field environments.

From these equations, a 2nd order finite volume Roe-approximate Riemann solver was derived[6]. Implemented with a multi-stage explicit update scheme, this scheme produced results that were validated and GDM simulations that were qualitatively correct. However, the large GDM magnetic fields of 10s to 100s of Tesla produced wave speeds a few percent the speed of sound. When trying to resolve GDMs down to the centimeter scale, this produced unsuitably small explicit time steps resulting in extremely slow convergence rates. The only evident solution to this problem is an implicit algorithm which has less restrictive stability constraints.

\section{Implicit Flow Solution}

A general finite volume implicit formulation with $\mathbf{U}, \mathbf{U}_{*}, \mathbf{F}$, and $\mathbf{S}$ being the state vector, a neighboring cell's state vector, a flux function, and a source term vector, respectively, is

$$
\begin{aligned}
\delta_{t} \mathbf{U}^{n+1} & +\frac{1}{V} \sum_{\text {faces }}\left(\frac{\partial \mathbf{F}^{n}}{\partial \mathbf{U}^{n}} \delta_{t} \mathbf{U}^{n+1}+\frac{\partial \mathbf{F}^{n}}{\partial \mathbf{U}_{*}^{n}} \delta_{t} \mathbf{U}_{*}^{n+1}\right) \cdot \hat{\mathbf{n}} d s \\
& -\frac{d \mathbf{S}^{n}}{d \mathbf{U}^{n}} \delta_{t} \mathbf{U}^{n+1}=-\frac{1}{V} \sum_{\text {faces }} \mathbf{F}^{n} \cdot \hat{\mathbf{n}} d s+\mathbf{S}^{n} .
\end{aligned}
$$

However, using the divergence theorem, you can obtain the source term as surface integral to get

$$
\mathbf{S}=-\frac{1}{V} \sum_{\text {faces }} \frac{\mathbf{B}_{x}+\mathbf{B}_{x *}}{2}\left(\begin{array}{c}
0 \\
\mathbf{B} \\
\mathbf{u} \\
\mathbf{u} \cdot \mathbf{B}
\end{array}\right) d s
$$

Incorporate this into the implicit formulation.

$$
\begin{aligned}
\delta_{t} \mathbf{U}^{n+1} & +\frac{1}{V} \sum_{\text {faces }}\left(\left(\frac{\partial \mathbf{F}^{n}}{\partial \mathbf{U}^{n}}-\frac{\partial \mathbf{S}^{n}}{\partial \mathbf{U}^{n}}\right) d s \delta_{t} \mathbf{U}^{n+1}+\right. \\
& \left.\left(\frac{\partial \mathbf{F}^{n}}{\partial \mathbf{U}_{*}^{n}}-\frac{\partial \mathbf{S}^{n}}{\partial \mathbf{U}_{*}^{n}}\right) d s \delta_{t} \mathbf{U}_{*}^{n+1}\right) \\
& =-\frac{1}{V} \sum_{\text {faces }} \mathbf{F}^{n} \cdot \hat{\mathbf{n}} d s+\mathbf{S}^{n}
\end{aligned}
$$

To determine the derivative of the flux function, start with a coordinate generalized Roe flux function. For simplicity, it is assumed that the flux direction is aligned with the $\hat{\mathbf{n}}$ vector is $(1,0,0)$. $\mathbf{K}$ is defined as a transformation matrix that converts from simulation coordinates $(\hat{x}, \hat{y}, \hat{z})$ to the flux normal coordinates $\left(\hat{n}, \hat{\tau}_{1}, \hat{\tau}_{2}\right)$ where the $\hat{\tau} \mathrm{s}$ are perpendicular axes,

$$
\begin{aligned}
& \mathbf{F}\left(\mathbf{U}_{L}, \mathbf{U}_{R}, \mathbf{K}\right)= \\
& \mathbf{K}^{-1}\left(\frac{1}{2}\left(\mathbf{F}_{L}\left(\mathbf{K} \mathbf{U}_{L}\right)+\mathbf{F}_{R}\left(\mathbf{K} \mathbf{U}_{R}\right)\right)\right. \\
& \left.-\frac{1}{2}\left|\mathbf{A}\left(\mathbf{K} \mathbf{U}_{L}, \mathbf{K} \mathbf{U}_{R}\right)\right|\left[\mathbf{K} \mathbf{U}_{R}-\mathbf{K} \mathbf{U}_{L}\right]\right)
\end{aligned}
$$

Taking the derivative of the flux function gives

$$
\begin{aligned}
\frac{\partial \mathbf{F}}{\partial \mathbf{U}_{L}} & =\mathbf{K}^{-1}\left(\frac{1}{2} \frac{d \mathbf{F}_{L}\left(\mathbf{K U}_{L}\right)}{d \mathbf{U}_{L}}\right. \\
& -\frac{1}{2}\left(-\left|\mathbf{A}\left(\mathbf{K} \mathbf{U}_{L}, \mathbf{K} \mathbf{U}_{R}\right)\right| \mathbf{K}\right. \\
& \left.\left.+\frac{\partial\left|\mathbf{A}\left(\mathbf{K} \mathbf{U}_{L}, \mathbf{K} \mathbf{U}_{R}\right)\right|}{\partial \mathbf{U}_{L}}\left[\mathbf{K} \mathbf{U}_{R}-\mathbf{K} \mathbf{U}_{L}\right]\right)\right)
\end{aligned}
$$

The term, $\frac{\partial\left|\mathbf{A}\left(\mathbf{K U}_{L}, \mathbf{K} \mathbf{U}_{R}\right)\right|}{\partial \mathbf{U}_{L}}$, is truly ugly. However, since we intend to use the derivative of the flux function in a matrix that will be assumed to be constant as part of a linear system, we can also assume that this term is zero.

$$
\frac{\partial \mathbf{F}}{\partial \mathbf{U}_{L}}=\mathbf{K}^{-1}\left(\frac{1}{2} \frac{d \mathbf{F}_{L}\left(\mathbf{K U}_{L}\right)}{d \mathbf{U}_{L}}+\frac{1}{2}\left|\mathbf{A}\left(\mathbf{K U}_{L}, \mathbf{K U}_{R}\right)\right| \mathbf{K}\right)
$$

Using $\frac{d \mathbf{F}_{L}\left(\mathbf{K U}_{L}\right)}{d \mathbf{U}_{L}}=\frac{d \mathbf{F}_{L}\left(\mathbf{K U}_{L}\right)}{d \mathbf{K} \mathbf{U}_{L}} \frac{d \mathbf{K U}_{L}}{d \mathbf{U}_{L}}=\mathbf{A}_{L}\left(\mathbf{K} \mathbf{U}_{L}\right) \mathbf{K}$, we get the following 


\section{References}

$$
\frac{\partial \mathbf{F}}{\partial \mathbf{U}_{L}}=\mathbf{K}^{-1}\left(\frac{1}{2} \mathbf{A}_{L}\left(\mathbf{K U}_{L}\right)+\frac{1}{2}\left|\mathbf{A}\left(\mathbf{K U}_{L}, \mathbf{K} \mathbf{U}_{R}\right)\right|\right) \mathbf{K}
$$

$\frac{\partial \mathbf{F}}{\partial \mathbf{U}_{R}}$ is derived the same way, and the derivative of the source terms is relatively simple. The actual derivative for the MHD system of equations described above is too extensive to print here, but their calculation is straight forward, if tedious.

Convert the implicit formulation to $\mathbf{A x}=\mathbf{b}$ form where $\mathbf{Q}$ is the vector of state vectors $\mathbf{U}$.

$$
\begin{aligned}
{\left[\mathbf{I}+\frac{\partial \mathcal{F}^{n}}{\partial \mathbf{Q}^{n}}-\frac{\partial \mathbf{S}^{n}}{\partial \mathbf{Q}^{n}}\right] \delta_{t} \mathbf{Q}^{n+1} } & =\delta_{t} \mathbf{Q}^{n} \\
& =-\operatorname{Residual}\left(\mathbf{Q}^{n}\right)
\end{aligned}
$$

To approach 2nd order accuracy, the residual of the current time step on the right hand side of the equation is calculated using the 2 nd order explicit scheme from the original work.

With the matrix $\mathbf{A}$ and vector $\mathbf{b}$, we must solved for the cell states of the next iteration. Since $\mathbf{A}$ is a large unstructered sparse matrix, this is extremely difficult. To tackle this system of equations, parallel computers and the Portable, Extensible Toolkit for Scientific Computing, PETSc[7, 8, 9], parallel library is used.

\section{Conclusions and Future Work}

A magnetohydrodynamic Roe-approximate finitevolume, 3D Cartesian adaptive scheme has been modified to address the extreme magnetic fields found in proposed GDM designs. Embedded magnetic fields are included to avoid the errors introduced by the large magnetic terms in the equations. Most recently, an implicit form of the scheme has been developed to address the time step limitations imposed by near relativistic wave speeds and resolution requirements on the order of centimeters.

Preliminary results from the new scheme match the output of the previously validated explicit code. The implicit algorithm needs to be rigorously validated, after which it can be applied to exploring the design issues of gas dynamic mirrors.
[1] Kammash, T. and Lee, M.-J., "Gasdynamic Fusion Propulsion System for Space Exploration", Journal of Propulsion and Power 11, 3, pp. 544$53,1995$.

[2] Kammash, T., Lee, M.-J., and Poston, D.I., "High-Thrust-High-Specific Impulse Gasdynamic Fusion Propulsion System", Journal of Propulsion and Power 13, 3, pp. 421-7, 1997.

[3] Kammash, T. and Galbraith, D.L., "Improved Physics Model for the Gasdynamic Fusion Propulsion System", Journal of Propulsion and Power 14, 1, pp. 24-8, 1998.

[4] Ohlandt, C. J., Kammash, T., and Powell, K. G., "Computational magnetohydrodynamic model of a avanced fusion fuel gas dynamic mirror propulsion system," AIAA Paper 2002-3932, AIAA/ASME/SAE/ASEE Joint Propulsion Conference and Exhibit, 38th, Indianapolis, IN, July 7-10, 2002.

[5] Ohlandt, C. J., Kammash, T., and Powell, K. G., "Computational magnetohydrodynamic model of a gasdynamic mirror propulsion system", AIAA Paper 99-2702, AIAA/ASME/SAE/ASEE Joint Propulsion Conference and Exhibit, 35th, Los Angeles, CA, June 20-24, 1999.

[6] Powell, K.G., Roe, P. L., Linde, T. J., Gombosi, T. I., and De Zeeuw, D. L., "A Solution-Adaptive Upwind Scheme for Ideal Magnetohydrodynamics", Journal of Computational Physics, 153, pp. 284-309, 1999.

[7] Balay, S., Gropp, W., McInnes, L. C., and Smith, B. F., "PETSci home page", http://www.mcs.anl.gov/petsc, 2001.

[8] Balay, S., Gropp, W., McInnes, L. C., and Smith, B. F., "PETSci Users Manual", ANL-95/11 - revision 2.1.2, Argonne National Laboratory, 2002.

[9] Balay, S., Gropp, W., McInnes, L. C., and Smith, B. F., "Efficient management of parallelism in object oriented numerical software libraries," Modern Software Tools in Scientific Computing, ed. Arge, Bruaset, and Langtangen, pp. 163-202, 1997. 


\begin{tabular}{|c|c|c|c|c|}
\hline Fuel & Products & $\begin{array}{c}\text { Total } \\
\text { Energy }[\mathrm{MeV}]\end{array}$ & $\begin{array}{c}\text { Charged Particle } \\
\text { Energy }[\mathrm{MeV}]\end{array}$ & $\begin{array}{c}\text { Optimal Ignition } \\
\text { Temperature }[\mathrm{keV}]\end{array}$ \\
\hline$D-T$ & $n+{ }^{4} \mathrm{He}$ & 17.6 & 3.5 & 10.5 \\
$D-D$ & $p+T$ & 4.0 & 4.0 & 15 \\
& $n+{ }^{3} \mathrm{He}$ & 3.3 & .8 & 15 \\
$D-{ }^{3} \mathrm{He}$ & $p+{ }^{4} \mathrm{He}$ & 18.3 & 18.3 & 60 \\
${ }^{3} \mathrm{He}-{ }^{3} \mathrm{He}$ & $2 p+{ }^{4} \mathrm{He}$ & 12.9 & 12.9 & 1000 \\
$p-{ }^{11} \mathrm{~B}$ & $3{ }^{4} \mathrm{He}$ & 8.7 & 8.7 & 150 \\
\hline
\end{tabular}

Table 1: Table of fusion fuels with relevant parameters.

\section{Interplanetary Vehicle \\ Using a Gasdynamic Mirror Fusion Propulsion System}

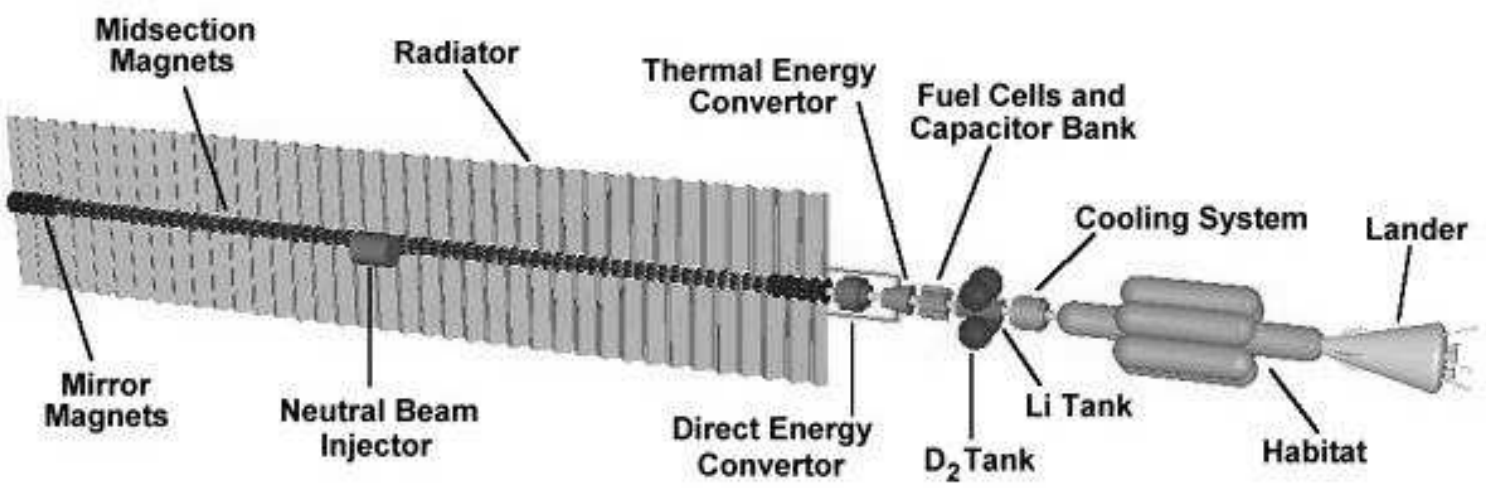

Figure 1: Interplanetary vehicle with a gas dynamic mirror fusion propulsion system.

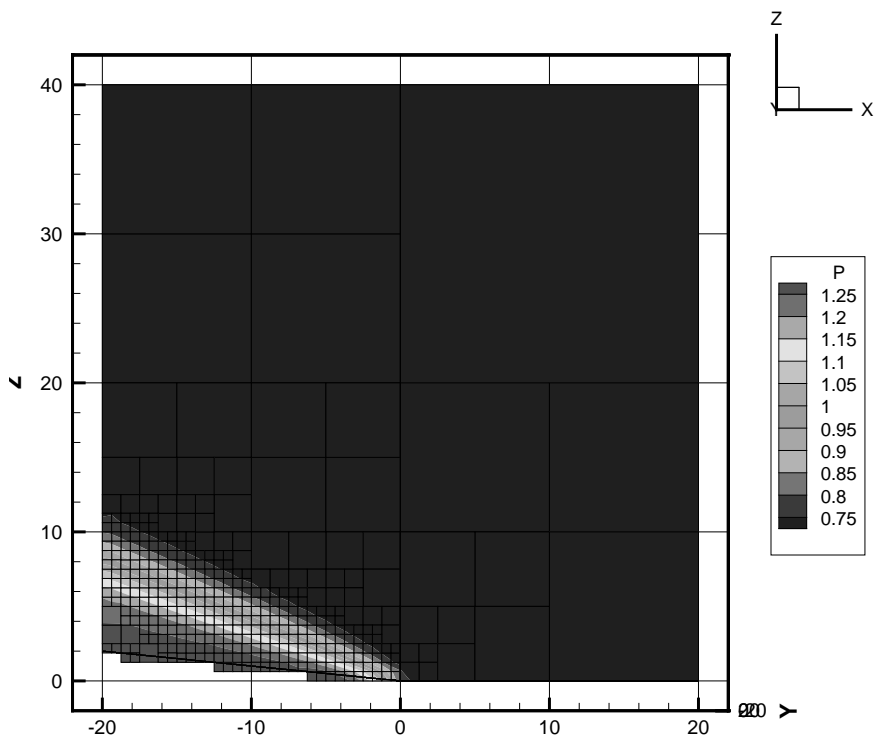

Figure 2: Sample result of a MHD shock wave over a wedge. 